\title{
Intracranial subdural hemorrhage following closed neural tube defect repair: illustrative case
}

\author{
Stacey Podkovik, DO, ${ }^{1}$ Jonathon Cavaleri, MD, ${ }^{2}$ Carli Bullis, $M D,{ }^{3}$ and Susan Durham, $M D^{3}$ \\ ${ }^{1}$ Department of Neurosurgery, Riverside University Health System, Riverside, California; ${ }^{2}$ Department of Neurosurgery, University of Southern California, Los Angeles, \\ California; and ${ }^{3}$ Department of Neurosurgery, Children's Hospital of Los Angeles, Los Angeles, California
}

\begin{abstract}
BACKGROUND Intracranial subdural hematomas (SDHs) due to intracranial hypotension after pediatric spine surgeries are an uncommon pathology. Such findings have typically been associated with intraoperative durotomies that are complicated by a subsequent cerebrospinal fluid (CSF) leak.

OBSERVATIONS The patient is a 17-year-old boy with a complex past medical history who received an uncomplicated S1-2 laminectomy for repair of his closed neural tube defect (CNTD), cord untethering, and resection of a lipomatous malformation. He returned to the hospital with consistent headaches and a 2-day history of intermittent left-sided weakness. Imaging demonstrated multiple subdural collections without a surgical site pseudomeningocele.

LESSONS The case was unique because there have been no documented cases of acute intracranial SDH after CNTD repair. There was no CSF leak, and spine imaging did not demonstrate any evidence of pseudomeningocele. The authors believed that intraoperative CSF loss may have created enough volume depletion to cause tearing of bridging veins. In younger adolescents, it is possible that an even smaller volume may cause similar effects. Additionally, the authors' case involved resection of the lipomatous malformation and an expansile duraplasty. Hypothetically, both can increase the lumbar cisternal compartment, which can collect a larger amount of CSF with gravity, despite no pseudomeningocele being present.
\end{abstract}

https://thejns.org/doi/abs/10.3171/CASE21159

KEYWORDS spontaneous intracranial hypotension; cerebrospinal fluid leak; spontaneous subdural hematoma; lipomyelomeningocele; pachymeningeal enhancement; neural tube defect

Intradural spine surgeries are extremely common procedures within the fields of both adult and pediatric neurosurgery. Distant intracranial extraaxial hemorrhages are a known, but uncommon, complication after intradural spinal procedures. Most of these occurrences are typically due to an iatrogenic cerebrospinal fluid (CSF) leak leading to intracranial hypotension. ${ }^{1}$ The incidence of subdural hematoma (SDH) in the setting of intracranial hypotension is approximately $10 \%$. $^{1}$ However, the incidence of SDH after intradural spine surgeries is reported to be as low as $0.8 \%{ }^{2}$ Within the pediatric population, these rates are even lower, with insufficient reported cases to present accurate statistical incidences. We present the case of a 17-year-old boy who underwent a first-time closed neural tube defect (CNTD) repair and developed multiple intracranial SDHs with no clinical or radiographic evidence of a postoperative CSF leak.

\section{Illustrative Case}

The patient is a 17-year-old boy with a past medical history of VACTERL syndrome (vertebral defects, anal atresia, cardiac defects, tracheoesophageal fistula, renal anomalies, and limb abnormalities) who presented with progressive low back and lower extremity pain and changes in urological function. He had never undergone spinal imaging previously. Magnetic resonance imaging (MRI) demonstrated a CNTD with a lipomatous malformation (Figs. 1 and 2). An uncomplicated S1-2 laminectomy was performed for repair of his CNTD and spinal cord untethering. Typical precautions, such as keeping the patient in mild Trendelenburg position, placing a surgical patty in the cephalad portion of the open thecal sac, and refilling the thecal sac with warmed saline before final dural closure, were taken. A duraplasty with bovine pericardium dural substitute

ABBREVIATIONS CNTD = closed neural tube defect; CSF = cerebrospinal fluid; CT = computed tomography; EDH = epidural hematoma; $\mathrm{MRI}=$ magnetic resonance imaging; SDH = subdural hematoma.

INCLUDE WHEN CITING Published July 12, 2021; DOI: 10.3171/CASE21159.

SUBMITTED March 15, 2021. ACCEPTED March 23, 2021.

(C) 2021 The authors, CC BY-NC-ND 4.0 (http://creativecommons.org/licenses/by-nc-nd/4.0/). 


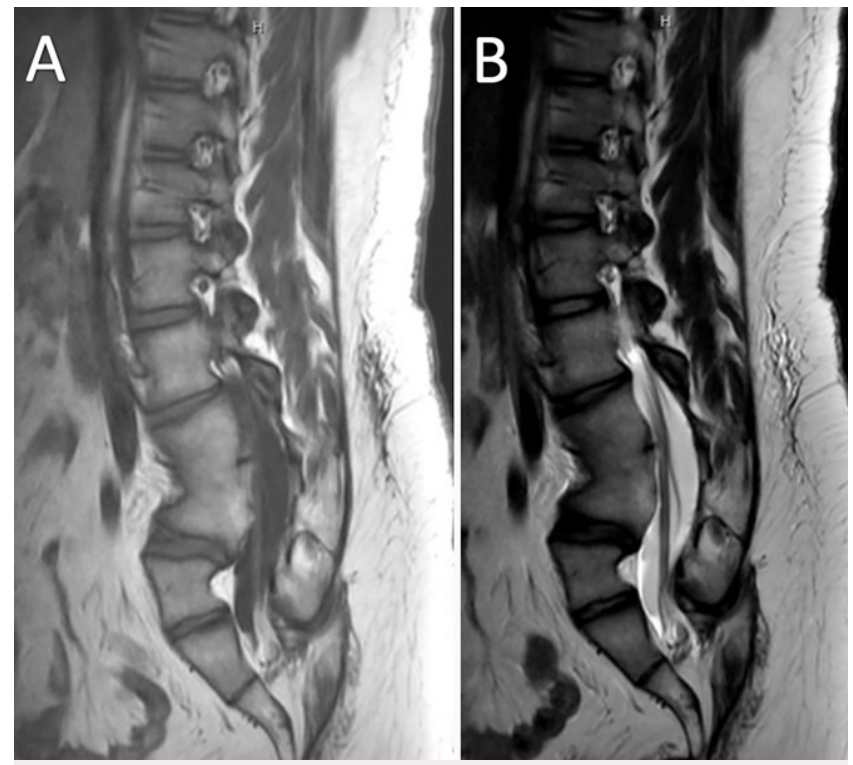

FIG. 1. Preoperative MRI of the lumbar spine. A: T1-weighted image demonstrating autofusion of the lower L3-4 vertebral bodies. B: T2weighted image demonstrating tethering of the spinal cord to the sacral spine.

was performed. A Valsalva maneuver to $30 \mathrm{~mm} \mathrm{Hg}$ did not demonstrate egress of CSF. Lastly, tissue sealant and bovine pericardium were placed over the duraplasty before proceeding with the remainder of the closure.

Starting on postoperative day 2, the patient began reporting mild headaches, which were not positional in nature. The patient was discharged home on postoperative day 3 , having met all criteria for discharge. Seven days after discharge, the patient returned with consistent headaches and a 2-day history of spontaneously occurring and resolving left-sided arm and leg weakness. Patient denied positional headaches, stating that they were improved with standing and ambulation, as well as any history of head trauma. On physical examination, the patient was neurologically intact with no evidence of focal neurological findings. During examination of his surgical site, there was no palpable pseudomeningocele, and the incision was healing well with no leak. Computed tomography (CT) and MRI demonstrated an acute 6-mm
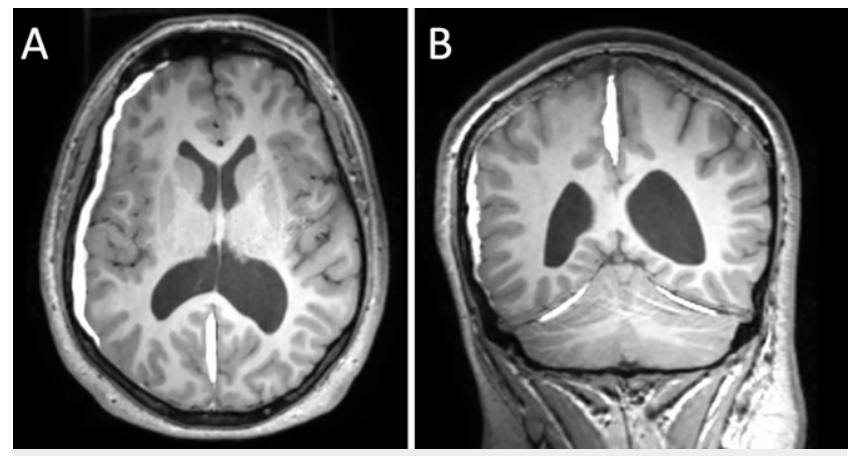

FIG. 2. T1 MRI on hospital readmission. A: Axial view demonstrating multiple SDH collections at the right convexity. B: Coronal view redemonstrating the convexity and parafalcine $\mathrm{SDH}$ as well as bilateral tentorial SDHs. right-sided convexity SDH with $2 \mathrm{~mm}$ of midline shift, a right parafalcine $\mathrm{SDH}$, and bilateral tentorial subdural collections (Fig. 2).

A lumbar spine MRI showed expected postoperative findings with no pseudomeningocele (Fig. 3).

The patient was managed nonsurgically with bedrest for 2 days and then was progressively sat up. He symptomatically improved, had no further episodes of left-sided weakness, and was subsequently discharged home with 2 weeks of prophylactic Keppra (UCB). At his 1month clinic follow-up, a repeat MRI of his head showed complete resolution of the subdural hemorrhages (Fig. 4).

\section{Discussion}

\section{Observations}

Spontaneous intracranial hypotension can be seen after various procedures, such as lumbar punctures, myelography, spinal anesthesia, ventriculo- and lumboperitoneal shunts, craniotomies, spine surgeries, and spine traumas. ${ }^{1-7}$ Patients commonly present with symptoms of orthostatic headaches, neck stiffness, nausea, vomiting, altered mentation, and possible seizures. ${ }^{3,8-13}$ Typically, symptoms present from 1 day to 1 week after the procedure, ${ }^{9,10}$ with $90 \%$ of headaches occurring within 3 days of the dural puncture or tear. ${ }^{1,10,14}$ These headaches are commonly described as occurring in frontal and occipital regions. ${ }^{15}$ In a retrospective review, Aoki analyzed postoperative complications of both ventriculoperitoneal and lumboperitoneal shunts and found that they had a $1.6 \%$ and $1 \%$ risk of chronic SDH complications, respectively. ${ }^{16}$ As it pertains to our patient, the presentation deviated from the stereotypical picture because he specifically denied any form of orthostatic headaches, with subjective improvement in symptoms upon standing and doing physical activity. Additionally, the most peculiar aspect of the patient's presenting symptoms was his episode of leftsided weakness that spontaneously resolved. Weakness that is due to compressive hematomas is usually persistent and does not have a waxing and waning quality. MRI and MR angiography during our workup did not demonstrate any evidence of diffusion restriction or vascular pathology, and an echocardiogram did not show evidence of any cardioembolic pathology. An alternative plausible explanation may have been focal seizures from cortical irritation caused by the $\mathrm{SDH}$, for which he was started on prophylactic Keppra.

Mokri presented a historical overview of the work done by both Alexander Monro and George Kellie in the 18th century regarding volumetric relationships between the intracranial components ${ }^{17}$ and how one cannot decrease without there being a concomitant increase in another. CSF volume depletion causes simultaneous caudal descent of the intracranial contents, which in turn leads to venous engorgement due to a negative pressure-like phenomenon. ${ }^{8}$ The downward tension on engorged venous vessels can lead to tearing of the veins and subsequent subdural hemorrhages. ${ }^{1,4,6,18-21}$ Additionally, the downward tension on dural venous sinuses, which are pain-sensitive, can contribute to headaches. ${ }^{4}$ Spontaneous CSF leaks that lead to intracranial pathology can be found in a certain subset of patients who have various connective tissue disorders; ${ }^{11,22}$ however, our patient demonstrated no past medical history concerning for any of these disorders.

Intracranial hypotension is a clinical diagnosis that can be corroborated with various imaging findings. When an $\mathrm{SDH}$ is present, both CT and MRI can aid in diagnosis of the intracranial pathology. In our institution, we prefer to obtain a fast-sequence "limited" MRI of the brain rather than a CT scan in children to avoid unnecessary radiation unless circumstances prohibit it. Aside from the intracranial bleeding that is readily visualized, contrast MRI can demonstrate a diffuse pachymeningeal enhancement. As vasculature becomes engorged in settings of intracranial hypotension, the pachymeninges 


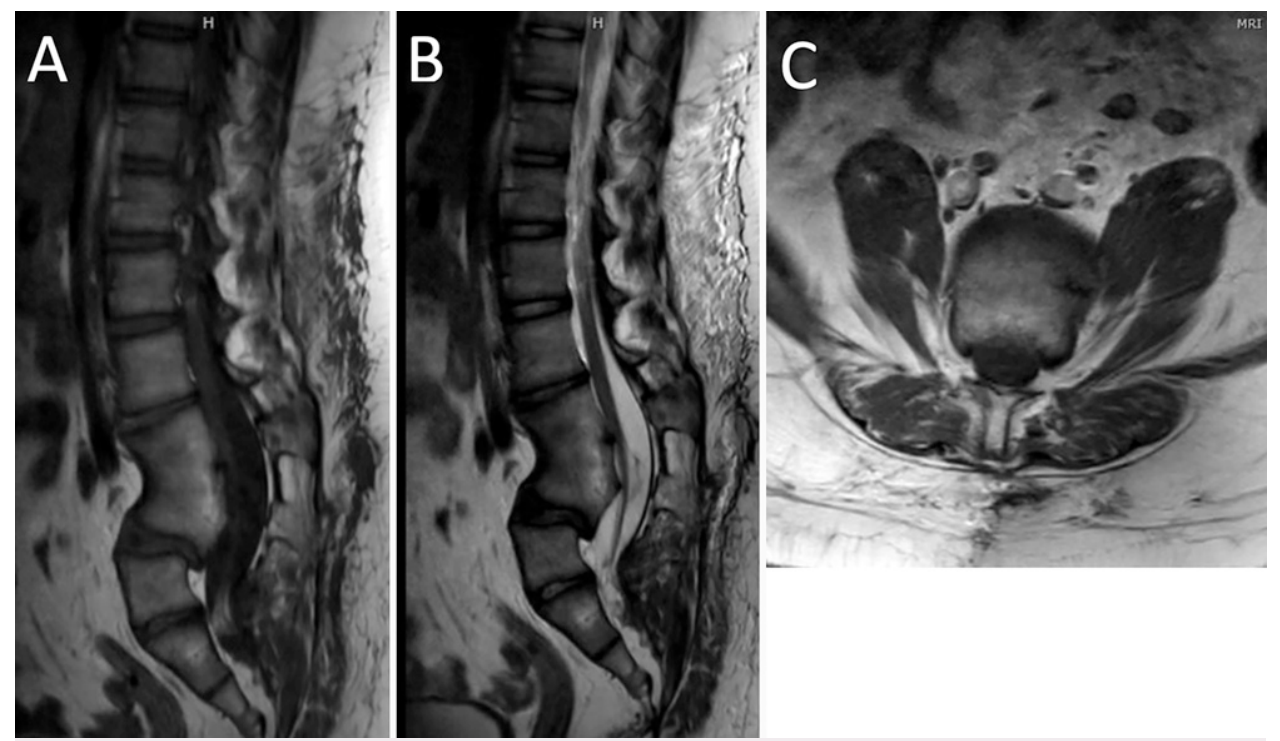

FIG. 3. Postoperative lumbar spine MRI. A: Sagittal $T 1$ image demonstrating autofusion of the lower $L 3-4$ vertebral bodies. B: Sagittal T2 image demonstrating no evidence of a pseudomeningocele. C: Axial T1 image without evidence of pseudomeningocele.

uptake gadolinium because of lack of a functioning blood-brain barrier. ${ }^{17}$ An additional finding involves the concept of "brain sag," which consists of flattening of the ventral pons and descent of the cerebellar tonsils. $^{4,8,12,13}$ Our patient demonstrated this characteristic finding (Fig. 5). Spine imaging is commonly also ordered to ensure that no obvious CSF leak exists at the site of the operative repair. MRI of the spine for our patient (Fig. 3) did not demonstrate any evidence of a pseudomeningocele or area of abnormal CSF egress.

\section{Lessons}

Upon thorough review of the literature, there are approximately four documented cases of epidural hematoma $(\mathrm{EDH})^{5}$ and four cases of postoperative SDH after intradural spinal surgery in pediatric patients. All four cases of EDHs received surgery for intradural tumors. ${ }^{5,20,23,24}$ Surash et al. noted that their case, a thoracic laminectomy for removal of a T4-5 intradural extramedullary metastatic medulloblastoma, was
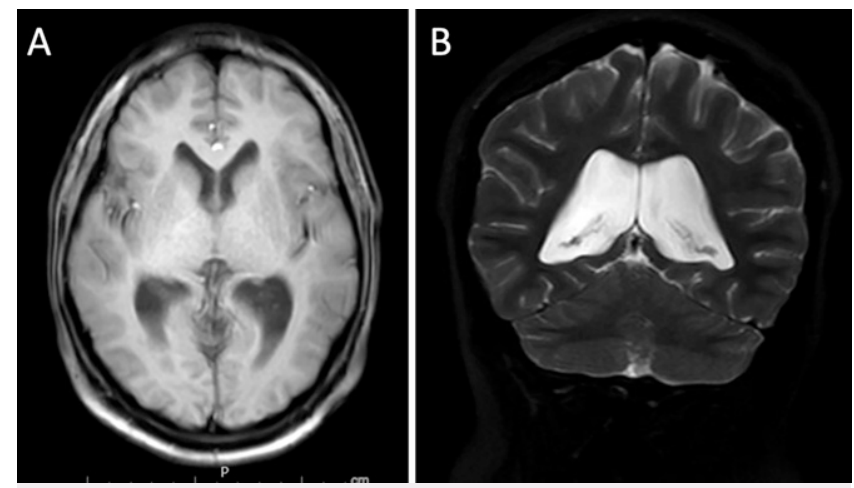

FIG. 4. A: Axial T1 MRI of the head demonstrating resolution of the right convexity SDH. B: Coronal T2 MRI of the head demonstrating resolution of the right convexity, parafalcine, and bilateral tentorial SDH. Unfortunately, a coronal $\mathrm{T} 1$ image was not obtained on the original sequencing and could not later be reconstructed. uncomplicated and did not have CSF egress after dural closure or postoperative CSF leak. They hypothesized that the bilateral postoperative $\mathrm{EDH}$ complication must have been due to intracranial hypotension caused by intraoperative CSF loss. ${ }^{23} \mathrm{Li}$ et al., who presented a 15year-old girl with bilateral EDH after a C4 intradural schwannoma resection, stated that children are more likely to form EDH from intracranial hypotension than adults because of weaker adhesions of their dura to the inner table of the skull. This theoretically leads to a potential space for dissection by the hematoma. ${ }^{20}$

In regards to SDHs from intracranial hypotension, Martínez-Lage et al. did a review of the literature and found a total of three pediatric patients who had a remotely located intracranial SDH after spine surgery. ${ }^{25}$ Of these three cases, only the one presented by Abel et al., which involved a 6-year-old girl who developed subdural hygromas after a spinal cord de-tethering procedure, was similar in terms of initial presenting pathology and the absence of any documented CSF leak postoperatively. ${ }^{9}$ Most recently, in 2018, Bhimani et al. reported on a 10 -year-old child who developed a right frontal SDH after undergoing
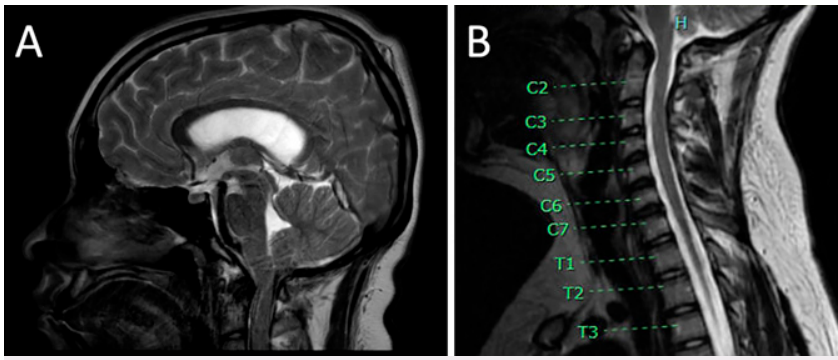

FIG. 5. A: MRI of the brain in the setting of postoperative headaches. Sagittal T2 image without contrast demonstrating crowding of the craniocervical junction with relative descent of the brainstem and cerebellar tonsils. B: Preoperative MRI of the cervical spine. Sagittal T2 image without contrast demonstrates a much more open craniocervical junction compared to the brain imaging in A. Unfortunately, no history of previous cranial imaging was found for comparison. 
scoliosis surgery and filum clipping because of a low-lying conus; her course was complicated by a postoperative CSF leak, which was noted after significant ( $300 \mathrm{~mL}$ over 3 days) surgical site drain output. $^{18}$

Our case is unique because there have been no documented cases of acute intracranial SDH after a CNTD repair. Additionally, there was no evidence of either an intraoperative or postoperative CSF leak, and spine imaging did not demonstrate any evidence of pseudomeningocele. In reviewing the previous cases, we believe that the patient's acute SDH may be attributed to two reasons. Initially, the intraoperative CSF loss may have created enough of a volume depletion to cause tearing of some of the bridging veins. Suess et al. found that after lumbar puncture procedures in adults, CSF leakage of approximately $240 \mathrm{~mL} /$ day could contribute to intracranial hypotension and its associated symptoms. ${ }^{26}$ In younger adolescents, it may be possible that an even smaller volume may cause similar effects. Additionally, our case involved repair of the lipomatous malformation at the end of the thecal sac, requiring the use of a duraplasty rather than a primary dural closure because of the absence of any suitable dura at the region of the lipomatous malformation. This could hypothetically increase the lumbar cisternal compartment, which can collect a larger amount of CSF with gravity, despite not having a pseudomeningocele present, although it was not noted on postoperative imaging.

Currently, the recommended treatment for someone with symptomatic intracranial hypotension without a hematoma causing substantial mass effect is keeping the head of the bed flat for at least 24 hours and administering analgesics, hydration, and caffeine..$^{1,6,10,22}$ Our patient was treated nonsurgically with bedrest for 48 hours before being sat up and ambulated. He symptomatically improved and was discharged home with close follow-up, including repeat imaging that showed subsequent resolution of his SDH. Epidural blood patches have also been proposed as a treatment if symptoms do not resolve with conservative treatment or in cases of a clear CSF leak, but they were not indicated in our patient's case.

Distant intracranial hemorrhage after spine surgeries in pediatric patients is an uncommon entity. We present a 17-year-old boy with primary repair of a CNTD that was complicated by an acute SDH without any evidence of postoperative CSF leakage. The patient was managed nonsurgically, with resolution of his symptoms and hematomas evident on repeat imaging. This case serves as an important lesson that although surgeons take great care to prevent postoperative CSF leaks, we also must be vigilant about how much CSF is lost during the controlled durotomy portion of the case because the momentary volume depletion can lead to future problems for the patient.

\section{References}

1. Chiravuri S, Wasserman R, Chawla A, Haider N. Subdural hematoma following spinal cord stimulator implant. Pain Physician. 2008;11(1):97-101.

2. Işik S, Yilmaz B, Ekşi MŞ, et al. Delayed onset intracranial subdural hematoma following spinal surgery. J Craniofac Surg. 2016;27(4):e370-e373.

3. Beier AD, Soo TM, Claybrooks R. Subdural hematoma after microdiscectomy: a case report and review of the literature. Spine J. 2009;9(10):e9-e12.

4. Jung YY, Ju Cl, Kim SW. Bilateral subdural hematoma due to an unnoticed dural tear during spine surgery. J Korean Neurosurg Soc. 2010;47(4):316-318.
5. Nemir J, Peterković V, Trninić I, et al. Intracranial epidural haematoma following surgical removal of a giant lumbosacral schwannoma: a case report and literature review. Pediatr Neurosurg. 2018;53(2):89-93.

6. Nowak R, Maliszewski M, Krawczyk L. Intracranial subdural hematoma and pneumocephalus after spinal instrumentation of myelodysplastic scoliosis. J Pediatr Orthop B. 2011;20(1):41-45.

7. Pavlin DJ, McDonald JS, Child B, Rusch V. Acute subdural hematoma: an unusual sequela to lumbar puncture. Anesthesiology. 1979;51(4):338-340.

8. Mokri B. Cerebrospinal fluid volume depletion and its emerging clinical/imaging syndromes. Neurosurg Focus. 2000;9(1):e6.

9. Abel TJ, Chowdhary A, Gabikian P, et al. Spontaneous subdural fluid collections following transection of a fatty filum terminale: case report and review of the literature. Pediatr Neurosurg. 2007;43(6):507-511.

10. Sciubba DM, Kretzer RM, Wang PP. Acute intracranial subdural hematoma following a lumbar CSF leak caused by spine surgery. Spine (Phila Pa 1976). 2005;30(24):E730-E732.

11. Podkovik S, Kashyap S, Bonda S, et al. Spontaneous intracranial hypotension: case study and review of the literature. Cureus. 2020;12(2):e7018.

12. Burkhard PR, Duff JM. Bilateral subdural hematomas following routine lumbar diskectomy. Headache. 2000;40(6): 480-482.

13. Ahmed SV, Jayawarna C, Jude E. Post lumbar puncture headache: diagnosis and management. Postgrad Med J. 2006;82(973): 713-716.

14. Reynolds F. Dural puncture and headache. BMJ. 1993;306(6882): 874-876.

15. Zakaria AF, Tsuji M. Intracranial subdural hematoma after lumbar spine surgery: a case report. Malays Orthop J. 2019;13(3): 85-87.

16. Aoki N. Lumboperitoneal shunt: clinical applications, complications, and comparison with ventriculoperitoneal shunt. Neurosurgery. 1990;26(6):998-1004.

17. Mokri B. The Monro-Kellie hypothesis: applications in CSF volume depletion. Neurology. 2001;56(12):1746-1748.

18. Bhimani R, Bhimani F, Singh P. Subdural hemorrhage after scoliosis and detethering of cord surgery. Case Reports in Medicine. Published online April 2, 2018. doi:10.1155/2018/5061898

19. Khalatbari MR, Khalatbari I, Moharamzad Y. Intracranial hemorrhage following lumbar spine surgery. Eur Spine J. 2012;21(10): 2091-2096

20. Li Z-J, Sun P, Dou Y-H, et al. Bilateral supratentorial epidural hematomas: a rare complication in adolescent spine surgery. Neurol Med Chir (Tokyo). 2012;52(9):646-648.

21. Watanabe A, Takai H, Ogino S, et al. Intracranial subdural hematoma after resection of a thoracic spinal cord tumor. J Spinal Disord Tech. 2002;15(6):533-536.

22. Schievink WI, Maya MM, Moser FG, Tourje J. Spectrum of subdural fluid collections in spontaneous intracranial hypotension. J Neurosurg. 2005;103(4):608-613.

23. Surash $S$, Bhargava $D$, Tyagi $A$. Bilateral extradural hematoma formation following excision of a thoracic intradural lesion. J Neurosurg Pediatr. 2009;3(2):137-140.

24. Ma X, Zhang $Y$, Wang $T$, et al. Acute intracranial hematoma formation following excision of a cervical subdural tumor: a report of two cases and literature review. Br J Neurosurg. 2014;28(1): $125-130$.

25. Martínez-Lage JF, López-Guerrero AL, Piqueras C, et al. Intracranial hemorrhage following surgery for occult spinal dysraphism: a case-based update. Childs Nerv Syst. 2015;31(6): 837-842. 
26. Suess O, Stendel R, Baur S, et al. Intracranial haemorrhage following lumbar myelography: case report and review of the literature. Neuroradiology. 2000;42(3):211-214.

\section{Disclosures}

The authors report no conflict of interest concerning the materials or methods used in this study or the findings specified in this paper.

\section{Author Contributions}

Conception and design: Podkovik, Bullis, Durham. Acquisition of data: Podkovik, Durham. Analysis and interpretation of data: Podkovik, Cavaleri, Durham. Drafting the article: Podkovik, Cavaleri. Critically revising the article: all authors. Reviewed submitted version of manuscript: all authors. Approved the final version of the manuscript on behalf of all authors: Podkovik. Administrative/technical/material support: Podkovik. Study supervision: Podkovik, Durham.

\section{Correspondence}

Stacey Podkovik: Riverside University Health System, Riverside, CA. s.podkovik@ruhealth.org. 